

\title{
Die „Torgauischen Gestüte" im 17. und 18. Jahrhundert
}

\author{
Steffen Bothendorf
}

Schloss Graditz Foto: Jens Ziegenbalg
Die Stadt Torgau verweist auf eine wechselvolle Historie und schrieb ein Stück der Geschichte des Kurfürstentums Sachsen unter den Wettinern mit. Graditz gehörte zunächst dem Zisterzienserkloster Dobrilugk (Doberlug). Nach Auflösung des Klosters 1541 gelangte der Hof an die Wettiner. Ab dieser Zeit begann die Pferdezuchtgeschichte von Graditz. Die Nähe der „Stutterey“ zur ehemaligen sächsischen Residenzstadt erforderte und erleichterte die Pferdehaltung. Das Ufer der Elbe mit seinem ertragsreichen Grünland bot sicher die Grundlage der Futterversorgung der Zuchtpferdebestände. Weitere Grundvoraussetzungen für Gestütsanlagen allgemein sind andererseits das Klima und die Möglichkeit der Großräumigkeit.

Das „Stutterey-Vorwerk“ Graditz fand bereits 1630 sowie 1637 im Zusammenhang mit dokumentierten Plünderungen während des Dreißigjährigen Krieges Erwähnung. In den Staatsbesitz gelangte die Anlage während der Regierungszeit des sächsischen Kurfürsten Johann Georg III. (1647-1691), der auch die Gründung des Hofgestüts Graditz veranlasste. Er schuf mit seiner aktiven Militärpolitik das erste stehende Heer in Sachsen und hatte demzufolge auch einen sehr hohen Bedarf an Pferden, Kutschpferden und Remonten (dreijährige Wallache und Stuten). Des- halb existierten bereits vor Graditz im Kurfürstentum Sachsen die Hofgestüte Bleesern bei Wittenberg (gegründet um 1509), Merseburg (gegründet 1563) und Kalkreuth bei Großenhain (gegründet 1588). Neben Graditz, als dessen Gründungsjahr 1686 gilt, entstanden um Torgau die Gestüte Repitz, Kreischau, Döhlen und später auch Neu-Bleesern. Da die Torgauer Bürger und Ratsherren sich nicht mit den Plänen ihres Kurfürsten einverstanden erklärten, versuchten sie, die Abgabe ihrer Grundstücke in die kurfürstliche Hand $\mathrm{zu}$ verhindern. Gegen ihren Willen kam es zur Vereinigung der Flächen, welche die Grundlage der „Torgauschen Gestüte“ ausmachten.

Unter Kurfürst Friedrich August I. (1670-1733) entwickelte sich Kursachsen zu einem der bedeutendsten, wirtschaftlich stärksten Länder Deutschlands. Somit ist es nicht verwunderlich, dass das Pferd neben seinem Einsatz im Militärwesen vor allem als Fortbewegungsmittel von großer Bedeutung war.

Kerstin Schöne recherchierte für ihre Dissertation die Gründe für die umfangreiche Pferdezucht in Graditz. In einer Akte heißt es: „Es hat das Land Sachsen jederzeit gute Pferde erzogen, die Arth daran ist anitzo fast ganz ausgegangen, nichts destoweniger hat es sowohl zur Equipage 




seiner königlichen Majestät Par force Jagdt, als zur Remontierung der Regimenter und Officier von der Armee gute Pferde von nöthen. Diese Pferde müssen anitzt alle außer Landes gekauft werden, und ist so viel Geld als darzu verwendet wird, für dem Lande verlohren."

Im Zusammenhang mit der Errichtung der „Torgauischen Gestüte“ beauftragte Friedrich August I. eine Kommission mit der Prüfung der örtlichen Gegebenheiten für die angedachten Gestütsanlagen. Nach den Vorstellungen des Kurfürsten sollten in den vier „Stuttereyn insgesamt“ 1.024 Pferde gehalten werden: in Döhlen 300, in Graditz und Kreischau je 240 und in Repitz 244 Pferde. Die staatliche Pferdezucht der damaligen Zeit hatte einen starken wirtschaftlichen Hintergrund und war nicht, wie so oft angenommen, der Eitelkeit der Herrscherhäuser geschuldet.

Zusammenfassend stellt sich die Entwicklung des sächsischen Hofgestütswesens im 17./18. Jahrhundert wie in nebenstehender Tabelle dar.

\section{Das sächsische Hofgestüt Graditz}

Einen ersten Hinweis zu Graditz finden wir in einer Urkunde des Jahres 1231, in welcher erwähnt wird, dass die Ileburger Grafen eine Erweiterung ins Gebiet östlich der Elbe vornahmen. Um das Jahr 1240 übernahmen die Mönche des Klosters Dobrilugk die Ortschaft vom Ritter Ulrich von Pack. Sie bauten Graditz 1241 zur Grangie (Klostervorwerk) aus. Da die Mönche das Handwerk der Landwirte und Tierzüchter verstanden, baute man Obst und Wein an und betrieb Fisch- und Pferdezucht. Die klösterliche Pferdezucht hatte Bestand, und man findet erstmals um 1630 Aufzeichnungen, in denen von

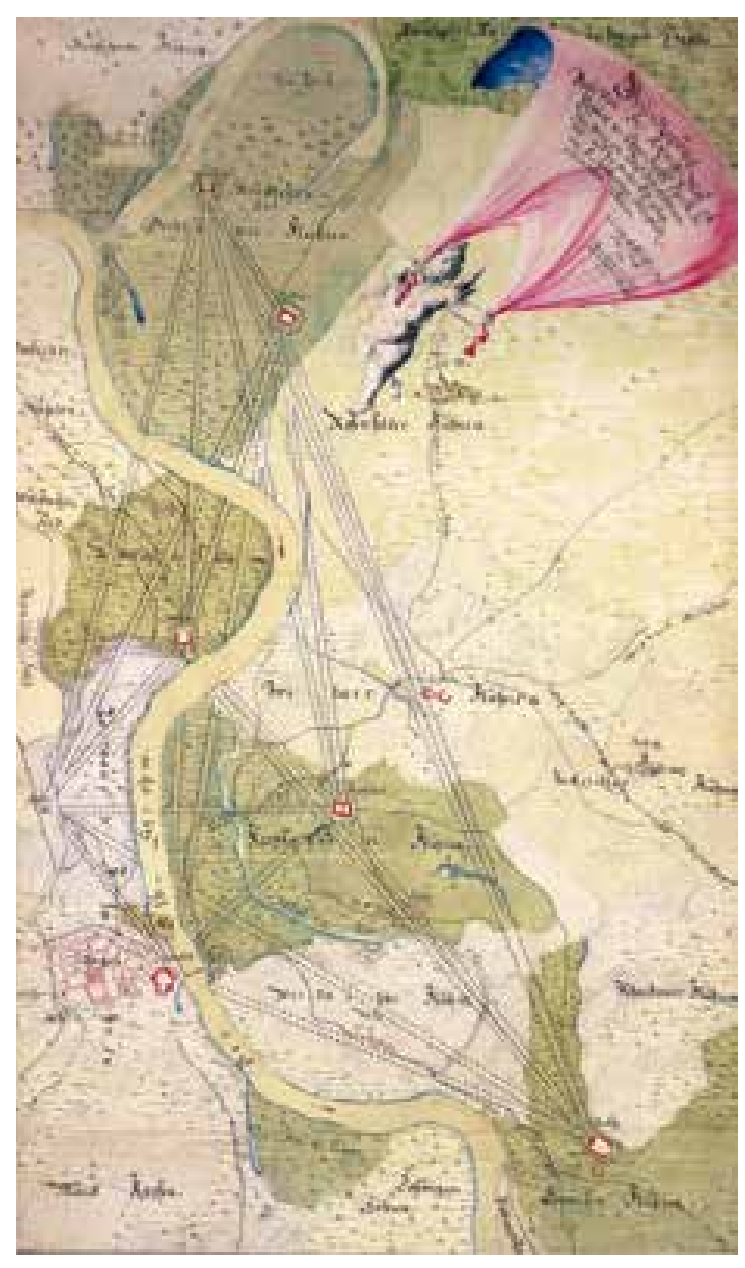

Übersichtsplan der „Torgauischen Gestüte“

Übersichtsplan zum Anlegen von Alleen zwischen dem Schloss Hartenfels in Torgau und den fünf "Stüttereyen", Hans Sigismund Ulrici, 1725 ○ Landesamt für Denkmalpflege Sachsen, Plansammlung

\begin{tabular}{|c|c|}
\hline 1665 & $\begin{array}{l}\text { Vorwerk Graditz ist kurfürstlich sächsisches Kammergut - } \\
\text { Pferdebestand } 26 \text { Stuten, } 2 \text { Beschäler und } 6 \text { Fohlen }\end{array}$ \\
\hline 1686 & Gründung des kurfürstlich sächsischen Hofgestüts Graditz \\
\hline 1686 & Einrichtung der „Stutterey“ in Repitz bei Torgau \\
\hline 1690 & Gründung des Vorwerks Döhlen \\
\hline 1692 & $\begin{array}{l}\text { Kammergut Kreischau wird als „Stutterey“ benannt - Pferdebestand } \\
12 \text { Hengste und } 50 \text { Mutterstuten }\end{array}$ \\
\hline 1695 & Blitzschlag und Brand zerstören ein Stallgebäude in Graditz \\
\hline 1716 & $\begin{array}{l}\text { Pferdebestand des kurfürstlich sächsischen Hofgestüts Bleesern bei } \\
\text { Wittenberg wird auf die Gestüte und Vorwerke Döhlen, } \\
\text { Kreischau und Repitz verteilt }\end{array}$ \\
\hline $1721 / 22$ & $\begin{array}{l}\text { Auflösung der kurfürstlich sächsischen Hofgestüte Kalkreuth bei } \\
\text { Großenhain, Bleesern bei Wittenberg, Zella bei Nossen, } \\
\text { Borschütz bei Mühlberg, Paudritzsch bei Leisnig, Repitz bei Torgau }\end{array}$ \\
\hline 1723 & $\begin{array}{l}\text { Zusammenschluss der Gestütshöfe und Flächen von Repitz, } \\
\text { Graditz, Döhlen, Neu-Bleesern und Kreischau unter der } \\
\text { Bezeichnung „die Torgauischen Gestüte“ }\end{array}$ \\
\hline 1729 & $\begin{array}{l}\text { Neuorganisation der sächsischen Gestütsverwaltung unter Land- } \\
\text { stallmeister Heinrich von Leipziger - Gesamtpferdebestand } \\
\text { der „Torgauischen Gestüte“ } 936 \text { Pferde, Gesamtflächenbestand der } \\
\text { „Torgauischen Gestüte“ ohne Kreischau 1.283,51 Hektar }\end{array}$ \\
\hline
\end{tabular}


Luftaufnahme des Hauptgestüts Graditz ๑ Wikimedia (Wolkenkratzer)



der Nutzung als „Stutterey“ geschrieben wurde. Kurfürst Friedrich August I. von Sachsen beauftragte den sächsischen Landbaumeister Matthäus Daniel Pöppelmann (1662-1736) mit der Planung und der Bauaufsicht der Graditzer Gestütsanlage, welche von 1721 bis 1726 gebaut wurde. Dem Bau der Stallungen folgte die Errichtung des Herrenhauses, später Landstallmeisterhaus oder Schlossgebäude genannt. Durch Errichtung des Torhauses im Jahr 1800 nach Plänen des Oberlandbaumeisters Christian Traugott Weinlig (1739-1799) wurde der Innenhof geschlossen.

\section{Das Gestüt Repitz}

Auf der Basis eines Gutachtens des Oberstallmeisters Johann Georg von Schleinitz und des Kammerrates Ludwig Gebhard von Hoym (1631-1711) vom 7. März 1686 wurde auch Repitz als Ort für

eine „Stutterey“ ausgewählt. Das Gutachten legte dar, dass sich das Gelände der Wüsten Mark Repitz als Standort für die Pferdezucht ideal eignet. In Repitz, gelegen am westlichen Ufer der Elbe, wurde am 10. Mai 1686 ein Gestüt gegründet. Zweckbestimmung der Gestütsanlage war die höfische Pferdezucht auf einer Fläche von 234 Hektar, davon 151 Hektar Wiesen und 72 Hektar Ackerland. Die Auswahl des Gestütsgeländes am Stadtrand von Torgau erwies sich als sehr gut, denn 1706 standen dann bereits 80 Mutterstuten in Repitz. Die Angliederung an die „Torgauischen Gestüte“ erfolgte im Jahre 1723. Der Gebäudekomplex, bestehend aus einem monumentalen Innenhof mit Torbogenhaus, wurde 1689 erbaut, was noch heute an der Giebelwand des östlichen Baues zu lesen ist. In der fortschreitenden Entwicklung des sächsischen Gestütswesens begann man mit der Bildung von Landbeschäleinrichtungen in Sachsen, darunter auch in Repitz.

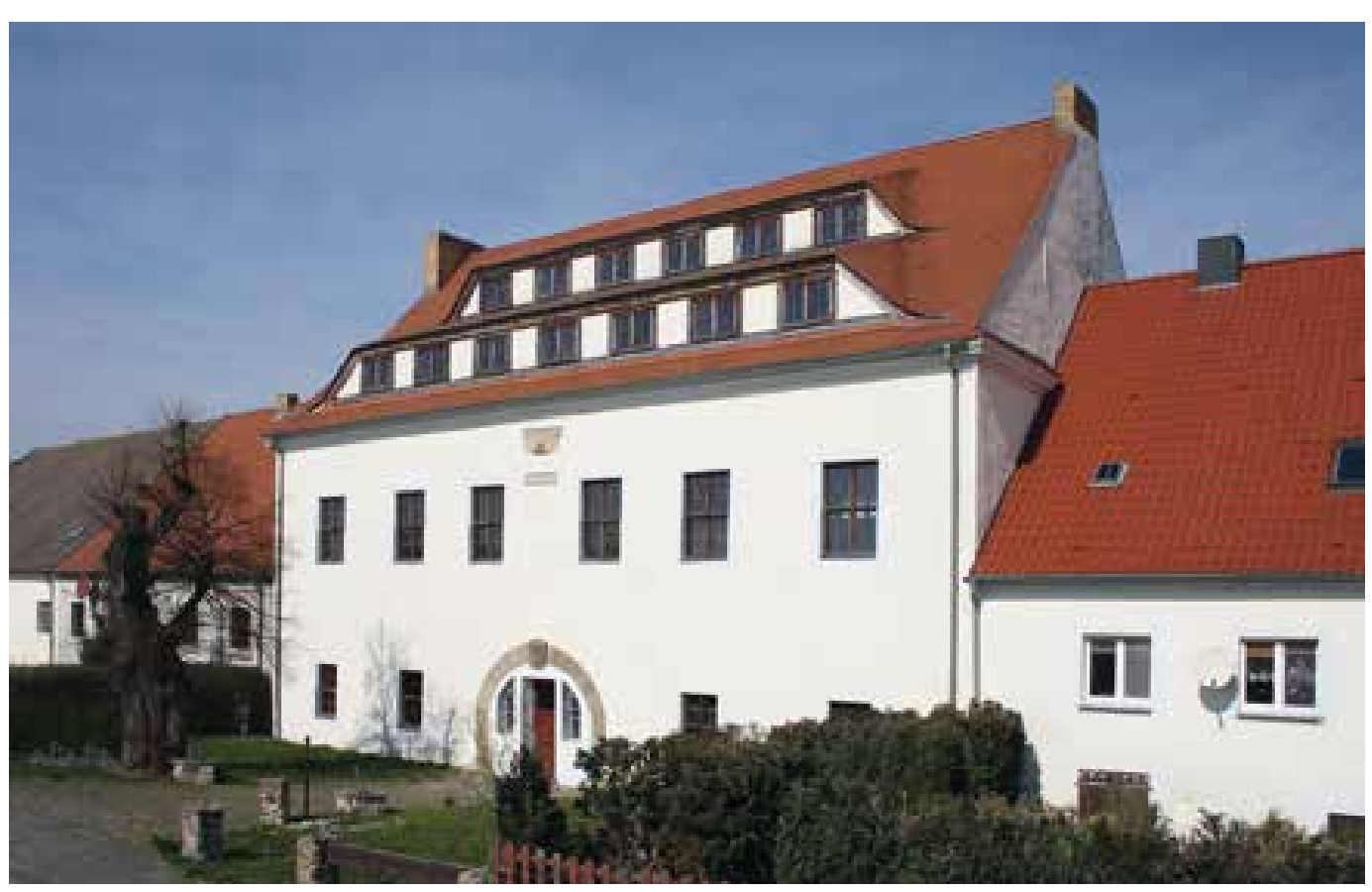




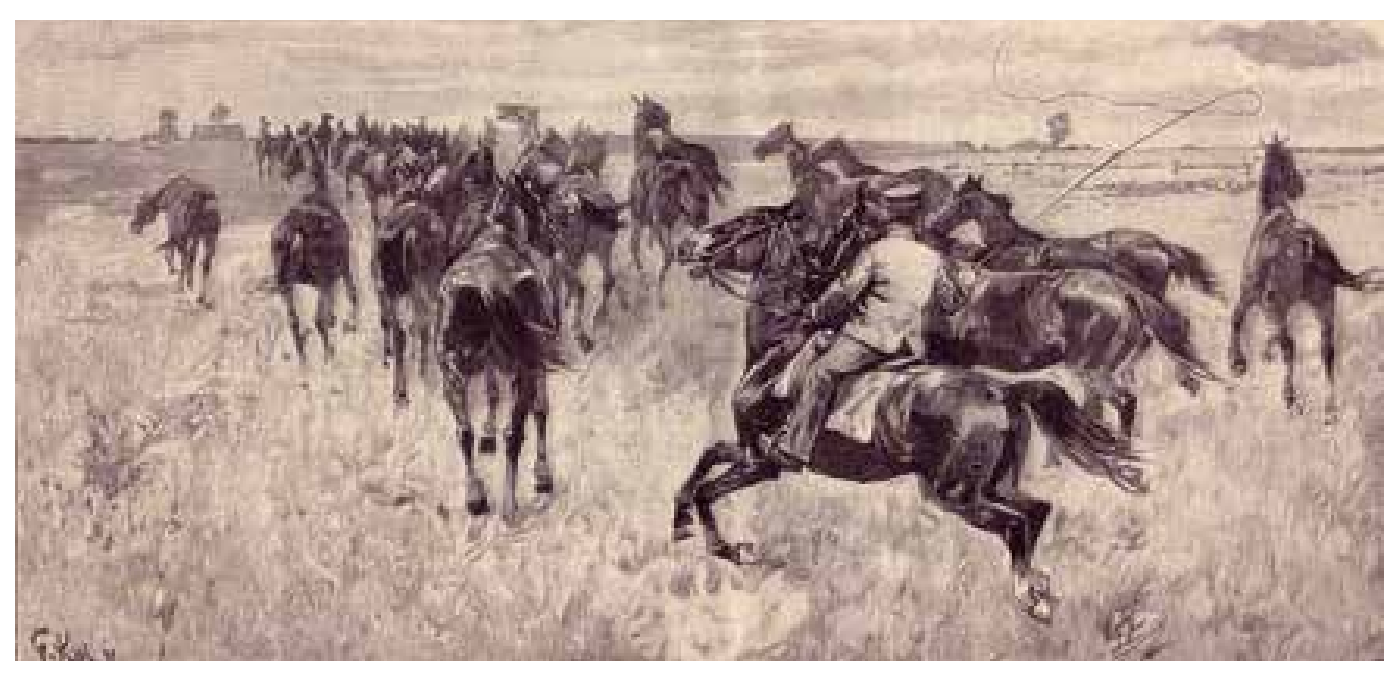

\section{Das Gestütsvorwerk Döhlen}

Der kleine Ort Döhlen, wie auch Graditz, Kreischau und Neu-Bleesern östlich der Elbe gelegen, wurde im Jahr 1339 erstmalig erwähnt. Nach verschiedensten historischen Zugehörigkeiten erwarb 1680 Kurfürst Johann Georg III. das Gestüt Döhlen. Die Pferdehaltung im Ort ist älter, und Döhlen war bereits vorher als „Stutterey“ bekannt. 1729 erfolgte unter Oberlandbaumeister Matthäus Daniel Pöppelmann eine Erneuerung der Anlage. Die „Stutterey Döhlen“ wird in den vorhandenen Archivbeständen immer im Zusammenhang mit Graditz genannt. Wahrscheinlich ist auch, dass, nach dem das Herrenhaus in Neu-Bleesern nicht gebaut werden durfte, die Verwaltung selbiger Anlage von Döhlen aus erfolgte. Bezogen auf das Pferdematerial liegt uns aus dem Jahr 1723 eine erste Bestandserfassung vor. Aus dieser geht hervor, dass im Gestütsvorwerk 17 Pferde standen.

\section{Das Gestütsvorwerk Kreischau}

Aus historischen Überlieferungen wissen wir, dass das Hauptgebäude in Kreischau 1692 errichtet wurde. Man baute es nach altem Vorbild als ein an vier Seiten geschlossenen Gestütshof aus. 1723 standen in Kreischau 141 Pferde. Mit der Übernahme des Vorsitzes der Kommission zur Errichtung der „Stuttereyen“ durch Kammerrat Georg Ludwig von Haxthausen änderte sich die Größe des Pferdebestandes. Da der Umfang der Weideflächen nicht ausreichte, verringerte er den ursprünglichen Pferdeetat auf unter 100 Pferde. Im weiteren Geschichtsverlauf wurde die „Stutterey Kreyschau“ aus den „Torgauischen Gestüten“ ausgegliedert und als Domäne genutzt.

\section{Das Gestütsvorwerk Neu-Bleesern}

Das 1721 eingerichtete Gestütsvorwerk Neu-Bleesern hat seinen Ursprung in Seegrehna, heute ein Ortsteil von Wittenberg. Dort, nahe Wittenberg, wurde 1487/88 von Kurfürst Friedrich dem Weisen (1463-1525) das Hofgestüt Bleesern gegründet. Oberlandbaumeister Wolf Caspar von Klengel (1630-1691) erneuerte die Gestütsanlage in der Mitte des 17. Jahrhunderts. Er schuf mit seiner Bau- weise das Grundkonzept aller nachfolgend im Kurfürstentum Sachsen gebauten Gestüte. Auch die Graditzer Gestütsanlage trägt Grundzüge Bleeserns und der barocken Bauweise der damaligen Zeit.

Am 23. März 1723 beauftragte Kurfürst Friedrich $\mathrm{Au}-$ gust I. von Sachsen den Landbaumeister Matthäus Daniel Pöppelmann, bei Torgau ein neues Gestüt unter dem Namen Neu-Bleesern zu errichten. Dieser konzipierte eine umfangreiche Gestütsanlage mit Herrenhaus für den Kurfürsten und König. Aus Sparsamkeitsgründen entstand das Gestüt dann jedoch in einer schlichteren Form. Man baute nur die Stallungen, nicht jedoch das Herrenhaus. Die Gestütsanlage verfügte über ein sehr ergiebiges Grünland von ca. 200 Hektar. Dieses nutzte das Hauptgestüt Graditz als Aufzuchtstätte der jungen Jahrgänge der Halbblutstuten und der Englischen Vollblutjährlingsstuten.



Die Gestüte um Torgau fielen 1815 an Preußen. Das Hauptgestüt Graditz und die kleineren Gestüte in der Nachbarschaft wurden als preußische Domänen weiterbetrieben. Das Hauptgestüt Graditz blieb ein wichtiger Standort für die Pferdezucht in Preußen. 1945 wurde die Domäne Graditz von der Provinzialverwaltung der Provinz Sachsen und später vom Land Sachsen-Anhalt übernommen. 1949 nahm man die Pferdezucht wieder auf. 1992 übernahm der Freistaat Sachsen das Hauptgestüt Graditz. Es untersteht einer gemeinsamen Gestütsverwaltung mit dem Landgestüt Moritzburg.
Stutenherde auf der Döhlener Flur, Stich, 19. Jahrhundert

Zum Weiterlesen

Steffen Bothendorf: Hauptgestüt Graditz - 325 Jahre Gestütspferdezucht. Moritzburg 2011. Siegfried von Lehndorff: HalbblutGestütbuch des Königlich Preussischen Hauptgestüts Graditz 1. Band. Berlin 1911.

Siegfried von Lehndorff: Ein Leben mit Pferde. Ein Beitrag zur Geschichte der Pferdezucht. Berlin/Paris 1943

Hans von Lehndorff: Menschen, Pferde, weites Land - Kindheitsund Jugenderinnerungen. München 1980.

Kerstin Schöne: Die Torgauer Gestütsbauten. Ein barocker Wirtschaftskomplex aus der Regierungszeit Augusts des Starken. Dissertation TU Dresden 1992. J. Schwartz: Das Königlich Preussische Hauptgestüt Graditz. Berlin 1870.

Matthias Donath: Schlösser und Herrenhäuser in Nordsachsen. Meißen 2014.

Gestüt Kreischau, ehemaliges Hauptgebäude Foto: Jens Ziegenbalg

\section{Autor}

Steffen Bothendorf Torgau 